\title{
MORE ON THE SCHUR GROUP OF A COMMUTATIVE RING
}

\author{
R.A. MOLLIN \\ Mathematics Department \\ University of Calgary \\ Calgary, Alberta, \\ T2N 1N4 Canada \\ (Received July 10, 1984)
}

ABSTRACT. The Schur group of a commutative ring, $R$, with identity consists of all classes in the Brauer group of $R$ which contain a homomorphic image of a group ring RG for some finite group $G$. It is the purpose of thjs article to continue an finvestigation of this group which was introduced in earljer work as a natural generalization of the Schur group of a field. We generalize certain facts pertaining to the latter, among which are results on extensions of automorphisms and decomposition of central simple algebras into a product of cyclics. Finally we introduce the Schur exponent of a ring which equals the well-known Schur index in the global or local field case.

KEY WORDS AND PHRASES. Schur index, group ring, automorphism.

AMS Subject classification 1979:

Primary 16 A26

1. INTRODUCTION.

All rings discussed herein will be assumed to be commutative wjth 1 . By an Azumaya $R$-algebra $A$ we will mean that $A$ is separable over $R$, faithful as an $R$-module and that $R \cdot 1$ coincides with the center of $A$. The symbol $B(R)$ wi.]l denote the $B r a u e r$ group of classes of Azumaya R-algebras. We refer the reader to De Meyer et al [1] for fundamental facts pertaining thereto.

The symbol $S(R)$ will refer to those classes [A] j.n $B(R)$ for which there exists a finite group $G$ and an $R$-algebra epimorphism from the group algebra $R G$ onto $A$. $S(R)$ is a subgroup of $B(R)$ called the Schur group of R. In De Meyer-Mo]lin [2] $S(R)$ was introduced and studied as a generalization of the Schur group of a field which has been extensively studied. As with the field case $S(R)$ was shown to be trivial when $R$ has non-zero characteristic. Therefore we assune henceforth that all rings have zero characteristic. It is worth noting by contrast that it was also proved in De Meyer-Mollin [2] that any finite Abelian group is the Schur group of some ring. The purpose of this paper is to further study $S(R)$. We require certain notation and concents, hawavi, before proceeding.

Let $S / R$ be a Galois extension of rings with group $G=G(S / R)$. We define a crossed product algebra made with $S, R$ and a 2-cocycle (factor set) $\beta: G \times G \rightarrow U(S)$, units of $S$, by $(S / R, \beta)=\sum_{\sigma \in G} S u_{\sigma}$. This is the Azumaya $R$-algebra which is free as 
as S-module on the basis $\left\{u_{\sigma}\right\}_{\sigma \in G}$ subject to the multiplication given by: $a u_{\sigma} b u_{\tau}=a b^{\sigma} \beta(\sigma, \tau)$ where $a, b \in S$ and $\sigma, \tau \in G$.

If $G=\langle\sigma\rangle$ is cyclic of order $n$ then $(S / R, \alpha)=\sum_{i=1} S u_{\sigma^{i}}$ denotes the

cyclic crossed product algebra in which $u_{\sigma}^{i}= \begin{cases}u_{\sigma^{i}} & \text { if } 1 \leq i<n . \\ \sigma & \text { if } i=n .\end{cases}$

We refer the reader to De Meyer et al [1] for further details.

Now let $\mathrm{R}$ be a connected ring; i.e. a ring whose only idempotents are 0 and 1 . We define a cyclotomic crossed product algebra as one of the form $(R[e(n)] / R, \beta)$ where $e(n)$ is a primitive $n^{\text {th }}$ root of unity which lies in the separable closure of $R$ and the values of $\beta$ are in the cyclic group generated by $e(n)$. If $R$ has finitely many idempotents then a cyclotomic R-algebra is the direct sum of cyclotomic algebras over the connected components of $R$. In De Meyer-Mollin [2] it was shown that the classes of $S(R)$ which contain a cyclotomic algebra form a subgroup which we denote by $S^{\prime}(R)$.

The Symbol $S^{\prime \prime}(R)$ will denote the subset of $S(R)$ consisting of those classes containing a homomorphic image of a separable group algebra RG. In De Meyer-Mollin [2] it was shown that if $R$ is an integrally closed Noetherian domain then $S^{\prime \prime}(R) \subseteq S^{\prime}(R)$. We conclude this section with some notation. $S(R, G)$ for a ring $R$ and a finite group $G$ will denote the subset of $S(R)$ consisting of those classes containing a homomorphic image of $R G$. Although $S(R, G)$ will serve mainly as a notational device herein, it is worth noting that, when $R$ is a field, Spiegel and Trojan [3] have investigated $S(R, G)$ from the point of view of determining when it is a group. $S^{\prime}(R, G)$ and $S^{\prime \prime}(R, G)$ are defined in an analagous fashion to that of $S(R, G)$. Finally $S^{\prime}(R, G),=S^{\prime}(R) \cap S(R, G)$ and $S^{\prime \prime}(R, G)=S^{\prime \prime}(R) \cap S(R, G)$.

2. AUTOMORPHISMS AND SUBGROUPS OF AZUMAYA ALGEBRAS.

The first result generalizes certain facts about the Schur group of an abelian number field to the Schur group of a Dedekind domain.

The first part of the theorem is a generalization of a standard fact (see Yamada [4]). The second part is a generalization of an "Amitsur-type" question; i.e. a question concerning subgroups of certain Azumaya algebras; which is an extension of a question related to Amitsur's classification of subgroups of division rings (see Amitsur [5]). In what follows exp [A] denotes the exponent of [A] in $B(R)$. Recall that all rings discussed herein have characteristic zero.

THEOREM 2.1 .

Let $G$ be a finite multiplicative group and let $R$ be a Dedekind domain. Suppose that $[A] \in S^{\prime}(R, G)$. Then:

(1) $\exp [A]$, divides $|G|$, and

(2) if $|G|$ is odd, A has no nontrivial idempotents and $R$ has no nontrivial odd order roots of unity then [A] is trivial and $G$ is cyclic.

PROOF :

Suppose that $K$ is the quotient field of $R$. Let $\left[A \phi_{R} K\right]=[A(X, K)]$ where $A(X, K)$ denotes the simple component of $K G$ corresponding to the absolutely irreducible character $X$ of $G$. Then by Herstein [6, Theorem 4.4.5, $p .119]$ we have that $\exp [A(X, K)]$ 
divides $m_{K}(X)$, the Schur index of $X$ over $K$, which in turn divides $|G|$ by Curtis et al [7, Theorem 27.11, p. 585]. Since $B(R) \rightarrow B(K)$ is one-to-one by De Meyer et a1 [1, Lemma 2.2, p. 136] then it follows that $\exp [A]$ divides $|G|$. This secures (1).

From De Meyer-Mollin [2] we have that if $\exp \left[A^{\dagger}\right]=n$ then $e(n)$ is in $R$. Hence $S(R)=S(R)_{2}$ the Sylow 2-subgroup of $S(R)$, since $R$ contains no nontrivial odd order roots of unity. However $|G|$ is odd which means $[A]=[R]$ by (1). Since $A$ has no nontrivial idempotents then $A$ has minimal rank in its class in $B(R) ; 1 . e . A=R$. But there is an R-algebra epimorphism $\phi: R G \rightarrow A$. Hence $\phi(G)$ is abelian. By Elgethun [8], $\phi(G)$ is a subgroup of of a division ring which implies that: all. Sylow subgroups of G are cyclic. by Amitsur [5]. Therefore G is cyclic. This completes the proof of theorem 1 .

The above result generalizes Mollin [9, Theorem 3.6, p. 243] to the ring case under the restriction that $G$ generates $A$. As pointed out in Elgethun [8], this restriction is necessary since subalgebras of arbitrary central separable algebras need not be separable over their centers.

In De Meyer-Mollin [2] it was proved that $S(R)$ (and $S^{\prime}(R)$ when $R$ has finitely many idempotents) are invariant subgroups of $B(R)$ under the natural action of Aut $(R)$ on $B(R)$. We describe this action at the algebra level since we need it for the following results which give information pertaining to extensions of automorphisms. If $[A] \in B(R)$ and $\sigma \in$ Aut $(R)$ then let $\sigma^{A}$ be the $R$-algebra equal to $A$ as a ring but with R-algebra action given by the rule $r * a=\sigma^{-1}(r)$ a for $r \in R$ and a $\in A$ with multiplication on the right being that in $A$.

THEOREM 2.2.

Let $T$ be a connected ring which is a finite Galois extension of a local ring $R$, with Galois group $G=G(T / R)$. Suppose $[B] \in B(T)$ and $B$ is maximally embedded in some R-Azumaya algebra $A\left(i . e . B=C_{A}(T)=\right.$ the centralizer of $T$ in $A$ ). Then $B$ is G-normal (i.e. every $\sigma \in G$ extends to Aut(B)). PROOF.

By Childs [10, Theorem 5.1, p. 13] we have $[B]=\left[C_{A}(S)\right]=\left[A^{\circ} \bigotimes_{R} S\right]$, where $A^{\circ}$ is the oppositive algebra of A. Now by Childs et a]. [11, Theorem 1.2, p. 26] we have that $\sigma \in G$ extends to an inner automorphism of $A^{\circ}$. Hence $\left[A^{\circ} \otimes_{R} S\right]=\left[A_{\sigma}^{\circ} \otimes_{R} S\right]=$ $\left.\int_{\sigma}\left(A^{\circ} \cdot \phi_{R} S\right)\right]$. Thus $[B]=\left[{ }_{\sigma} B\right]$ which implies that $B$ is G-normal by De Meyer [12]. This secures theorem 2.2.

Maintaining the hypothesis of the theorem we have the following result which is immediate from theorem 2 and De Meyer [12, Theorem 12, p. 335].

COROLLARY 2.3.

$B \equiv C{ }_{R} T$ for some $[C] \in B(R)$ if and only if $B$ is the kernel of the Teichmuller cocycle map, (See Childs [10]).

Corollary 2.3 leaves open the following question. Given [B] $\in S(T)$, when is [C] $\epsilon S(R)$ ? For fields and quaternion algebras this question was attacked in Mollin [13], where we asked the specific question: When are all quaternion algebras in the Schur group of an imaginary subfield of a cyclotomic field "induced" from the Schur group of its maximal real subfield. It is worth noting that it is well known that 
such quaternion algebras are induced from quaternion algebras in the Brauer group of the maximal real subfield.

We now turn to the question: If $[A] \in S^{\prime}(R)$ then when does $\sigma \in$ Aut (R) extend to Aut (A)?

In De Meyer-Mollin [2] it was proved that when $R$ is an integral domain then an element of order $n$ in $S^{\prime}(R)$ is fixed by an element $\sigma$ in Aut(R) if and only if $R$ contains $e(n)$ fixed by $\sigma$. When $R$ is a field of characteristic zero we can obtain more; namely that the following are equivalent (see Moliin [14] and [9])

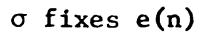

However the equivalence of (ii) and (iii) fails for rings in general. For rings, hrowever, we do have De Meyer [12, Lemma 1, p. 328] which gives us the equivalence of: (ii) and (iv): A is R-isomorphic to $\sigma^{A}$. Moreover an example in (De Meyer [12, p. 336] of a ring $R$ is given to demonstrate that we may have (iii) without (jv). Nevertheless we do have the following result.

PROPOSITION 2.4 .

Let $R$ be a regular local ring or a ring of polynomials in one variable over a perfect field. If $[A] \in S^{\prime}(R)$ and $A$ has exponent $n$ then the following are equivalent. (1) $\quad R$ contains $e(n)$ fixed by $\sigma \in$ Aut $(R)$

(2) $\sigma \in$ Aut (R) extends to Aut (A).

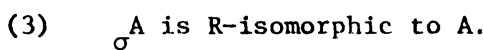

(4) $\quad[\mathrm{A}]=[\mathrm{A}]$.

PROOF

As remarked in the discussion preceeding the proposition, (2) and (3) are equivalent. Moreover, (3) and (4) are equivalent by De Heyer [12, Proposition ]3, p. 335]. Finally (1) and (4) are equivalent by De Meyer-Mollin [2], thereby securing the proposition.

COROLLARY 2.5 .

Suppose $T / R$ is an extension of regular Jocal rings with Galois group G. Suppose that [B] $\in S^{\prime}(T)$ with $\exp [B]$ - $n$ and $B$ is maximally embedded in some R-Azumaya algebra. Then $e(n)$ is in $R$.

PROOF

First we note that $e(n)$ is in $T$ by De Meyer-Mollin [2]. By theorem 2 every $\sigma \in G$ extends to Aut (B). Hence by proposition 4 every $\sigma \in G$ fixes e(n); 1.e.e(n) is in $R$. This completes the corollary.

3. DECOMPOSITION OF AZUMAYA ALGEBRAS.

One of the most important open questions in the theory of the Brauer group of a field $R$ is: Is $B(R)$ generated by cyclics? i.e. Are there integers $m$ and $n$ and cyclic algebras $A_{1}, \ldots, A_{r}$ such that for an R-division algebra $D$ with [D] $\in B(R)$ we have $M_{m}(D) \cong M_{n}(R) \diamond_{R} A_{1} \diamond_{R} \ldots s_{R} A_{r}$ where $M_{m}(*)$ denotes a full ring of $m \times m$ matrices over *. Recently, however Merkurjev and Suslin [15] were able to prove that if $D$ has exponent $t$, say, and $e(t)$ is in $R$, then $D$ is a product of cyclic algebras of exponent $t$, and in particular $D$ has an abelian splitting field. This 
result has special importance for $S(R)$ since we always have $e(t)$ in $R$ when there is an element of exponent $t$ in $S(R)$; 1 .e. the general question is answered by [15] for the Schur group of a field. Now, this does not mean that $D$ is isomorphic to a tensor product of cyclics. This was shown to be false in the early 1930's when A.A. Albert produced an example of a non-cyclic division ring of index and exponent 4 . Now we turn to the ring case and attack similar questions.

For the remainder of this section we assume that $S$ is a connected ring, finite Galois over $R$ with Abelian group $G=G(S / R)$, and such that the Picard group of $S$ is trivial. Thus we have $G \cong<\sigma_{1}>x \ldots .<<\sigma_{t}>$ where the cyclic group $<\sigma_{i}>$ has order $n_{1}$, say, for $i=1,2, \ldots, t$. Let $H_{i}=\prod_{j \neq i}<\sigma_{j}>=G\left(S / R_{i}\right)$; i.e. $R_{i}$ is the fixed ring of $H_{1}$.

Now let $B(S / R)$ be the kernel of the map $B(R) \rightarrow B(S)$ given by $[A] \rightarrow\left[A x_{R} S\right.$ ]. By De Meyer et al $\left[1\right.$, Corollary 1.3, p. 116] we have $B(S / R) \cong H^{2}(G, U(S)$ ), (the second cohomology group). Suppose now that $[A] \in B(S / R)$; then $A=(S / R, \beta)$ for some 2-cocycle $\beta$. A natural question to ask is: When does $A$ have a similar decomposition to that of $G$; i.e. as a tensor product of cyclic algebras $A_{i}$ where $\left[A_{i}\right] \in B\left(R_{1} / R\right)$ for $i=1,2, \ldots, t$ ? The following result provides an answer. We maintain the above notation and assumptions, and define a synmetric factor set $\beta$ on a group $G$ to be one such that $\beta(\sigma, \tau)=\beta(\tau, \sigma)$ for all $\sigma, \tau \in G$.

THEOREM 3.1.

Suppose that $G$ has order $n$ and exponent $m$ such that $n \in U(R)$ and $e(m) \in R$. If [A] $\in B(S / R)$ with $A=(S / R, \beta)$ then $A \cong A_{1} \diamond_{R} \ldots \diamond_{R} \Lambda_{t}$ such that $\left[A_{1}\right] \in B\left(R_{i} / R\right)$ if $\beta$ is a symmetric factor set. Conversely we have a weaker result; viz: If $[A]=\left[\begin{array}{lllll}A_{1} & \theta_{R} & \cdots & \Delta_{R} & A_{t}\end{array}\right]$ with $\left[A_{i}\right] \in B\left(R_{i} / R\right)$ then is $\beta$ cohomologous to a symmetric factor set.

PROOF

The proof follows exactly as in Mollin [16, Theorem 3.5] mutatis mutandis.

We note that the above generalizes the result for generic abelian crossed products over fields obtained in Amitsur et al [17, Lemma 1.5, p. 81].

Now maintaining the notation of the theorem we have the following result which yields a criterion for a decomposition of Schur group elements into a product of cyclics.

COROLLARY 3.2 .

Let $R$ be an integrally closed Noetherian domain. Suppose that $[A] \in S^{\prime \prime}(R, G)$ where $\exp [A]=\exp G=m$. Then $[A]=\left[A_{1} x_{R} \cdots s_{R} A_{t}\right]$ if and only if $\beta$ is cohomologous to a symmetric factor set. (Note that if $\beta$ is a symmetric factor set then we have the stronger result that $A: \because A_{1} \cdot s_{R} \ldots{ }_{R} A_{t} \cdot$ ) PROOF

Since RG is separable then the generalized Maschke theorem dictates that $|G| \in U(R)$. Since $R$ is an integrally closed Noetheprian domain then $S^{\prime \prime}(R) \subseteq S^{\prime}(R)$ from De Meyer-Mollin [2]. Moreover whenever there are elements of exponent $m$ in $S^{\prime}(R)$ then by De Meyer-Mollin [2] we have that $e(m)$ is in $R$. The result now follows from Theorem 3.1.

The hypothesis of Corollary 3.2 which requires $\exp [A]=|G(S / R)|$ where $A \cong(S / R, \beta)$ does not always hold of course. In fact the question pertaining to when 
such an $S$ exists is related (in the case where $R$ is a field) to the Brauer splitting theorem, which states that a finite group of exponent $m$ has $Q(e(m))$ as a splitting field where $Q$ is the field of rational numbers. In Szeto [18] this theorem has been generalized to the ring case, viz: If $G$ is a finite group of exponent $m$, and $R$ is a connected ring with RG being separable then $R[e(m)]$ is a splitting ring for G; $i . e$. $\mathrm{R}[\mathrm{e}(\mathrm{m})] \mathrm{G}$ is a direct sum of Azumaya algebras which are trivial in $\mathrm{B}(\mathrm{R}[\mathrm{e}(\mathrm{m})])$.

In the case where $R$ is an abelian extension of $Q$ we investigated in Mollin [19] and [20] conditions for the existence of a splitting field $S$ of $A$ where [A] $\in S(R),|S: R|=\exp [A]$ and $S \subseteq Q\left(e_{m}\right)$ where $m=\exp G$ with $A$ being a simple component of RG. An open area of inquiry is to obtain conditions for the existence of such an $S$ when $R$ is a connected ring, in view of the generalized Brauer theorem cfted above. Finally this generalized splitting theorem allows us to formulate an analogue of the Schur index.over a ring. The next section deals with this development.

4. THE SCHUR EXPONENT OVER A RING.

When $R$ is a field of characteristic zero and $[A] \in S(R)$ then $A$ is a simple component of RG for some finite group G. Moreover, if $X$ is an absolutely irreducible character of $G$ then $A(X, R) \cong M_{n}(D)$, a full ring of $n \times n$ matrices over a division ring $D$ and, $\sqrt{|D: R|}=m_{R}(R)$ is called the $\underline{\text { Schur }}$ index of $X$ over $R$. For an arbitrary ring of characteristic zero we do not have such a beautiful setup. However when we restrict to a connected ring and use the generalized Braver splitting theorem discussed in $\$ 3$ then we do have a fundamental layout which closely approximates the above.

Suppose $S$ is a connected ring and $S$ is a splitting ring for a finite group $G$; f.e. $S G=B_{1} \nrightarrow \ldots \cdot \theta B_{S} ;|G| \in U(S)$, a direct sum of central separable S-algebras whose classes are trivial in $B(S)$. In Szeto [18] and [21] characters $T(i)$ of. G are defined in such a way that they are in a one-to-one correspondence with the $B_{j}$. Moreover it is established that $\mathrm{T}^{(i)}(g)$ is a sum of $n_{i}$ th roots of unity where $g \in G$, $g^{n_{i}}=1$, and $S\left[T^{(i)}\right]$ is finitely generated, projective and separable over $S$.

Now, suppose that $R$ is a connected ring which does not split $G$, but $|G| \in U(R)$. Then $R G=A_{1} \rightarrow \ldots \rightarrow A_{t}$ where the $A_{j}$ are Azumaya over their centers. If $|G|=n$ and $R[e(n)] G=B_{1} \rightarrow \ldots$ !t) $B_{S}$ then let $T^{(i)}$ be the character of $G$ corresponding to $B_{i}$ and $B_{i}=R[e(n)] G E_{i}$ where $E_{i}$ is the $i^{\text {th }}$ primitive central idempotent (see Szeto [18]). We note that $R[e(n)]$ is Galois, hence abelian, over $R$ by Janusz [22, Proposition 2.6, p. 469]. Moreover, since $R\left[T^{(i)}\right] \subseteq R[e(n)]$ then $H_{i}=G\left(R\left[T^{(i)}\right] / R\right)$ is abelian.

Now we assume that $R$ is a regular domain. Therefore $B(R[e(n)]) \rightarrow B(K(e(n)))$ is injective. Hence for $\sigma \in \mathrm{H}_{i}$ we have $\mathrm{R}[\mathrm{e}(\mathrm{n})] \mathrm{GE}_{i}{ }^{\sigma} \rightarrow \mathrm{K}(\mathrm{e}(\mathrm{n})) \mathrm{GE}_{i}{ }^{\sigma}$. But $\mathrm{A}_{i} \otimes_{R} \mathrm{~K}=$ $A\left(X_{i}, K\right)$ and by Dornhoff [23, Lemma 24.7, p. 124] $A\left(X_{i}, K\right) s_{K}\left(X_{i}\right) K(e(n)) \cong K(e(n)) G E$ where the sum ranges over all $\sigma$ in $G\left(K\left(X_{i}\right) / K\right)$. Thus we have $A_{i}{ }^{\Delta} C_{i} R[e(n)]=$ $\sum_{\sigma \in H_{i}} R[e(n)] G_{i}^{\sigma}$, where $C_{i}$ is the center of $A_{i}$. Therefore we have demonstrated that each $A_{1}$ correxponds to exactly one $T^{(i)}$. Hence we may define the Azumaya algebra $A\left(T^{(i)^{i}}, R\right)$ corresponding to $T^{(i)}$. Let $m_{R}\left(T^{(i)}\right)$ be the exponent of $A(T(i), R)$ in $S^{\prime \prime}\left(C_{i}\right)$. Thus we define for a regular domain $R$ the Schur exponent of $T$ (i) over $R$; viz. $m_{R}\left(T^{(i)}\right)$. We note that this is a natural definition since in the case of a local or 
a global field the exponent equals the index of the representative division algebra, i.e. the Schur index. We note that since $R$ is a regular domain then $R$ is an integrally closed Neotherian domain. Therefore by De Meyer-Mollin [2] we have $\left[A\left(T^{(i)}, R\right)\right] \in S^{\prime \prime}\left(C_{i}\right) \subseteq S^{\prime}\left(C_{i}\right)$, i.e. as in the field case we are dealing with cyclotomic ilgebras.

We note that by theorem $2.1(1)$ we have that if $R$ is Dedekind domain then $m_{R}\left(T{ }^{(i)}\right.$ ) divides $|G|$ for $\left[A\left(T^{(i)}, R\right)\right] \in S\left(C_{i}, G\right)$. This generalizes the standard fact for the Schur group of a field. We leave the reader with the fact that it is an open question as to whether or not other standard facts pertaining to the Schur index gerteralize (see Yamada, 141).

AKNOWLEDGEMENT

This research is supported by N.S.E.R.C. of Canada.

\section{REFERENCE}

1. F.R. De Meyer and E. Ingraham, 'Separable Algebras Over Commutative Rings', Lecture Notes in Math. 181, Springer-Verlag, 1971.

2. F.R. De Meyer and R.A. Mollin, 'The Schur Group of a Commutative Ring', (to appear: J. Pure and Applied Algebra).

3. E. Spiegel and A. Trojan, 'Simple Compoisents in Group Algebras', J. Algebra, 83(1), (1983), 33-38.

4. 'T. Yamada, 'The Schur Subgroup of the Brauer Group', Lecture Note in Math. 397. Springer-Verlag 1974.

J. S.A. Amisur, 'Finite Subgroups of Division Rings', Trans. Amer. Math. Soc. 80 (1955), 361-386.

6. I.N. Herstein, 'Noncummutative Rings', Carus Monograph, No. 15, M.A.A. (1968).

7. C. Curtis and I. Reiner, 'Methods of Representation Theory' 1, Wiley Interscience (1981). 8. E.D. Elgethun, 'Subgroups of Central Separable Algebras', Canad. J. Math.
25 (4) (1973), 881-887.

9. R.A. Mollin, 'Herstein's Conjecture, Automorphisms and the Schur Group', Communication in Alg. 6 (3), (1978), 237-248.

10. L.N. Childs, 'On Normal Azumaya Algebras and the Teichmuller Cocycle Map', J. Algebra 23, (1972), 1-17.

11. L.N. Childs and F.R. De Meyer, 'On Automorphisms of Separable Algebras' Pacific J. Math. 23 (1), (1967), 25-34.

12. F.R. De Meyer, 'An Action of the Automorphism Group of a Commutative Ring On It's Brauer Group', Pacific J. Math., 97 (2), (1981), 327-338. 13. R.A. Mol1in, 'Induced Quaternion Algebras in the Schur Group', Canad. J. Math.
33 (6), 1981, 1370-1379. 14. R.A. Mollin, 'The Schur Group of a Field of Characteristic Zero', Pacific J.
Math. 76 (1978), 471-478.

15. A.S. Merkurjev and A.A. Suslin, 'K-Cohomology of Severi-Brauer Varieties and Norm Residue Homomorphism's (to appear D A H C C P).

16. R.A. Mollin, 'Admissible Groups, Symmetric Factor Sets, and Simple Algebras,

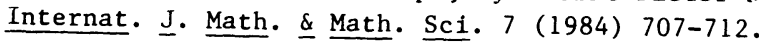


17. S.A. Amitsur and D. Saltman, 'Generic Abelian Crossed Products and p-Algebras' J. Algebra 51 (1978), 76-87.

18. G. Szeto, 'On the Brauer Splitting Theorem', Pacific J. Nath. 31 (2), (1969), 505-512.

19. R.A. Mollin, 'Minimal Cyclotomic Splitting Fields for Group Characters', (to appear, Proceedings A.M.S.)

20. R.A. Mollin, 'Splitting Fields and Group Characters', J. Reine Angew Math. 315, (1980), 107-114.

21. G. Szeto, 'The Group Character and Split Group Algebras', Pacific J. Math. 34 (1), (1970), 183-191.

22. G.J. Janusz, 'Separable Algebras Over Commutative Kings', Trans. Amer. Math. Soc. $122(1966)$, 461-479.

23. L. Dornhoff, 'Group Representation Theory', l'art. A, Dekker, 1971. 


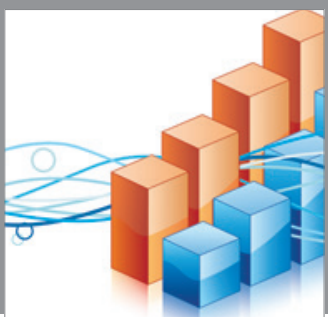

Advances in

Operations Research

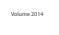

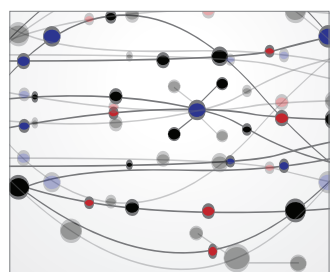

\section{The Scientific} World Journal
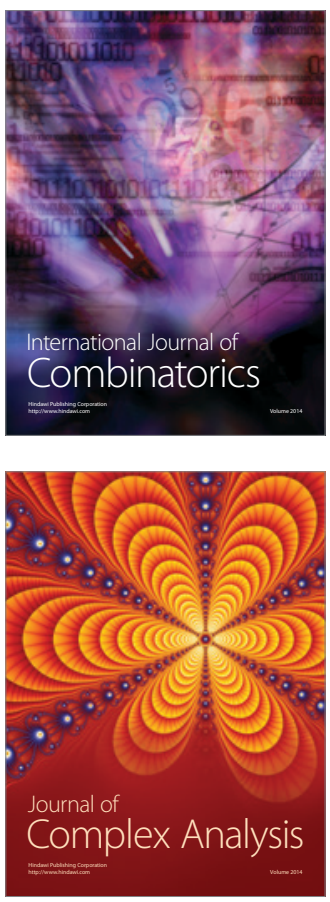

International Journal of

Mathematics and

Mathematical

Sciences
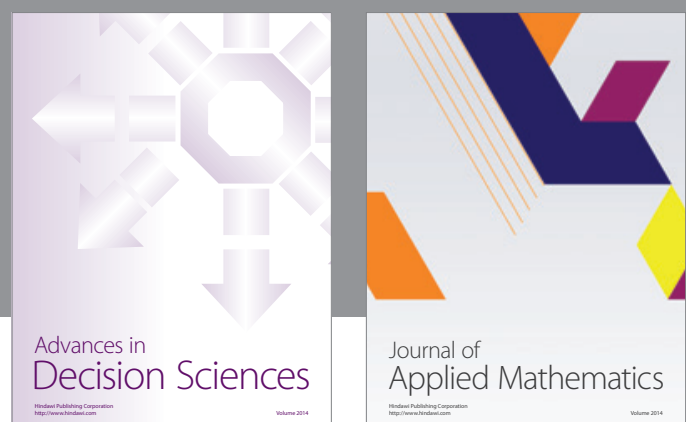

Journal of

Applied Mathematics
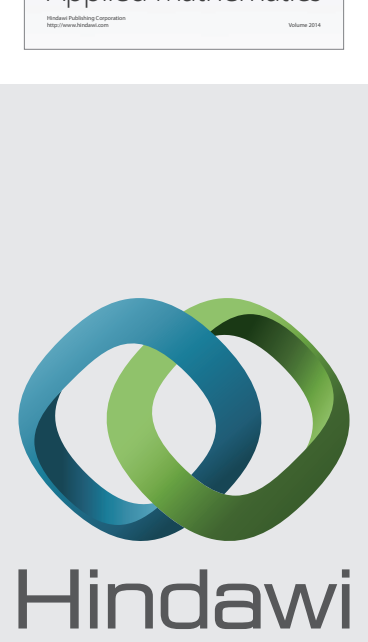

Submit your manuscripts at http://www.hindawi.com
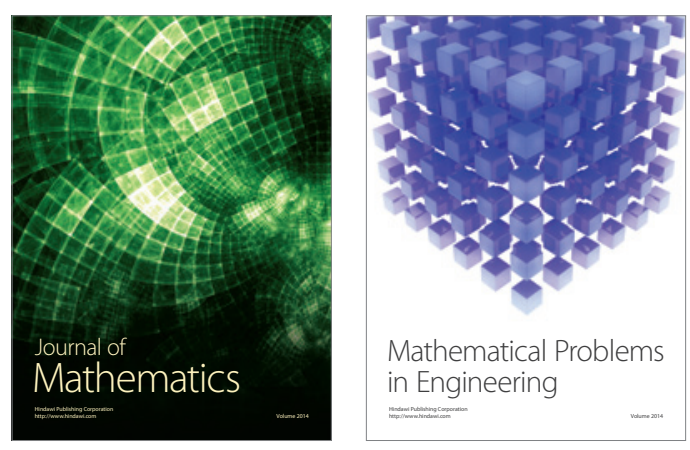

Mathematical Problems in Engineering
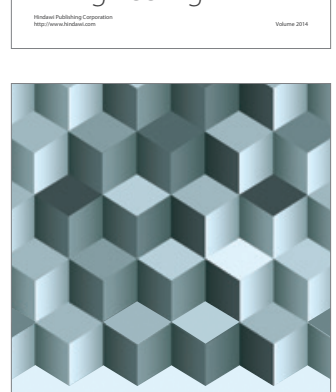

Journal of

Function Spaces
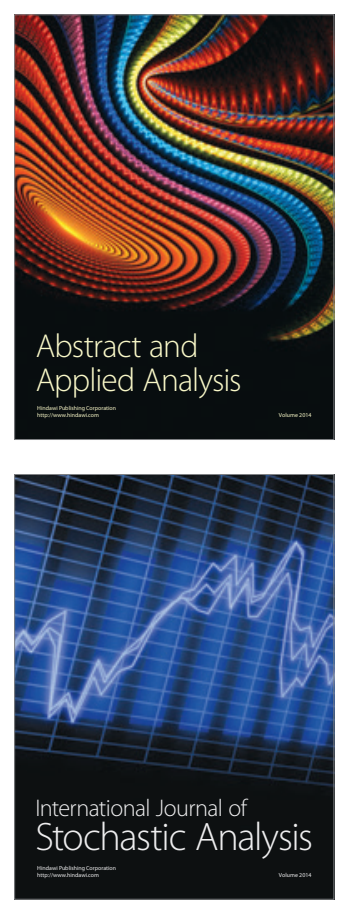

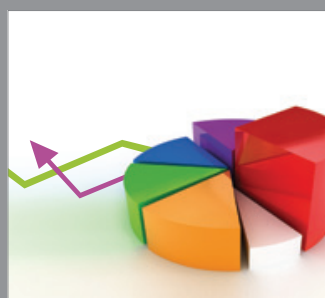

ournal of

Probability and Statistics

Promensencen
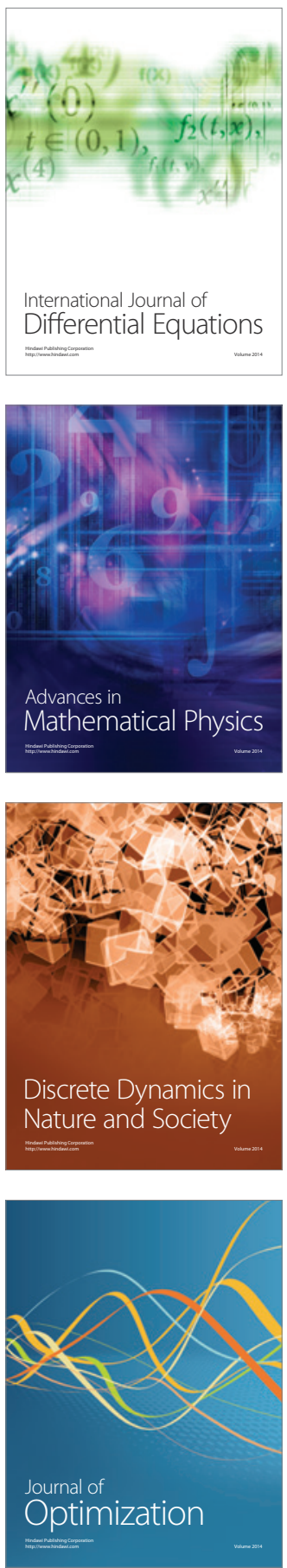